\title{
Synthetic antibody: prospects in aquaculture biosecurity.
}

\begin{abstract}
The emerging technology of aptamers that is also known as synthetic antibodies is rivalling antibodies research in the recent years. The unique yet important features of aptamers are advancing antibodies in diverse applications, which include disease diagnosis, prophylactic and therapeutic. The versatility of aptamer has further extended its application to function as gene expression modulator, known as synthetic riboswitches. This report reviewed and discussed the applications of aptamers technology in the biosecurity of aquaculture, the promising developments in biosensor detection for disease diagnosis as well as prophylactic and therapeutic measurements. The application of aptamers technology in immunophenotyping study of aquatic animal is highlighted. Lastly, the future perspective of aptamers in the management of aquatic animal health is discussed, special emphasis on the potential application of aptamers as synthetic riboswitches to enhance host immunity, as well as the growth performance.
\end{abstract}

Keyword : Synthetic antibody; Aptamers; Disease diagnosis; Therapeutic; Prophylactic; Immunophenotyping; Synthetic riboswitches. 